\title{
Some Optimum and Suboptimum Frame Synchronizers for Binary Data in Gaussian Noise
}

Nielsen, Palle Tolstrup

Published in:

I E E E Transactions on Communications

Publication date:

1973

Document Version

Publisher's PDF, also known as Version of record

Link back to DTU Orbit

Citation $(A P A)$ :

Nielsen, P. T. (1973). Some Optimum and Suboptimum Frame Synchronizers for Binary Data in Gaussian Noise. I E E E Transactions on Communications, 21(6), 770-772.

\section{General rights}

Copyright and moral rights for the publications made accessible in the public portal are retained by the authors and/or other copyright owners and it is a condition of accessing publications that users recognise and abide by the legal requirements associated with these rights.

- Users may download and print one copy of any publication from the public portal for the purpose of private study or research.

- You may not further distribute the material or use it for any profit-making activity or commercial gain

- You may freely distribute the URL identifying the publication in the public portal

If you believe that this document breaches copyright please contact us providing details, and we will remove access to the work immediately and investigate your claim. 


\section{CONCLUSION}

In this correspondence the route to obtaining natural moments from Walsh characteristic functions for continuous functions has been considered. The result of $(20)$ is the main conjecture presented. At the time, the merits of this result remain mathematical. And in relevance to this, the derivation of a relation (23) for Walsh transforms of dyadic derivatives was made.

\section{REFERENCES}

[1] J. Pearl, "Application of Walsh transform to statistical analysis," IEEE Trans. Syst., Man, Cybern., vol. SMC-1, pp. 111-119, Apr. 1971.

[2] N. J. Fine, "Generalized Walsh functions," Trans. Amer. Math. Soc., vol. 69 , pp. $66-77,1950$.

[3] F. E. Weiser, "Walsh functions analysis of instantaneous nonlinear stochastic problems," Ph.D. dissertation, Poly technic Inst. Brooklyn, Brooklyn, N.Y., 1964.

[4] M. Maqusi, "Walsh functions and the sampling principle," presented at the 1972 Walsh Functions Symp., Naval Res. Lab., Washington, D.C.

[5] M. Maqusi and"L. C. Ludeman, "Output dyadic correlation functions of instantaneous nonlinear devices," presented at the 1972 Nat. Electron. Conf., Chicago, III.

[6] F. Pichler, "Walsh functions and linear system theory," presented at the 1970 Walsh Functions Symp., Naval Res. Lab., Washington, D.C.

[7] S. O. Rice, "Mathematical analysis of random noise," Bell Syst. Tech. J., vols. 23 and 24 , para. 4.8, 1944 and 1945.

[8] J. E. Gibbs, "Sine waves and Walsh waves in physics," presented at the 1970 Walsh Functions Symp., Naval Res. Lab., Washington, D.C.

[9] H. F. Harmuth, Transmission of Information by Orthogonal Functions. Berlin: Springer-Verlag, 1969.

[10] N. J. Fine, "On the Walsh functions," Trans. Amer. Math. Soc., vol. 65 , pp. 372-414, 1949.

\section{Some Optimum and Suboptimum Frame Synchronizers for Binary Data in Gaussian Noise}

\section{P. TOLSTRUP NIELSEN}

Abstract-In this correspondence we investigate the performance of several optimum and suboptimum devices for locating a sync word in data corrupted by Gaussian noise. One suboptimum synchronizer, which is extremely simple to instrument, is shown to perform virtually optimally over the entire range of interesting signal-to-noise ratios.

\section{INTRODUCTION}

In a recent paper [1], Massey derived the optimum decision rule for locating a sync word in data corrupted by additive white Gaussian noise. Here we report additional results on the performance of the optimum synchronizer and we demonstrate that a simplified decision rule, which is extremely easy to implement, yields a performance that for all signal-to-noise ratios of practical interest is virtually indistinguishable from that of the optimum synchronizer.

We consider the situation where an $L$-b sync word $s=\left(s_{0}\right.$, $s_{1}, \cdots, s_{L-1}$ ) is inserted periodically with period $N$ in a random binary data stream. Each $s_{i}$ is either +1 or -1 . The bit intervals are assumed known at the receiver and the input to the synchronizer is a span of $N$ consecutive outputs from the detector $r=\left(r_{0}, r_{1}, \cdots, r_{N-1}\right)$. If the first bit of the sync word is received as $r_{m}$ we have the relation

$$
r=\sqrt{E} T^{m}(s d)+n
$$

where $d=\left(d_{L}, d_{L+1}, \cdots, d_{N-1}\right)$ consists of $N-L$ independent

Paper approved by the Communication Theory Committee of the IEEE Communications Society for publication without oral presentation. Manuscript received August 28, 1972.

The author is with the Laboratory for Communication Theory, Technical University of Denmark, Lyngby, Denmark. random data bits for which $\operatorname{Pr}\left\{d_{i}=1\right\}=\operatorname{Pr}\left\{d_{i}=-1\right\}=\frac{1}{2}$, and $T(\cdot)$ is the cyclic shift operator defined by $T(s d)=\left(d_{N-1}\right.$, $\left.s_{0}, \cdots, s_{L-1}, d_{0}, \cdots, d_{N-2}\right)$. The noise contributions $n_{i}$ are independent zero-mean Gaussian random variables with variance $N_{0} / 2$. The parameter to be estimated $m$ is a priori equally likely to be anywhere in the interval $0 \leqslant m \leqslant N-1$.

Massey [1] derived the following rule for obtaining the smallest-probability-of-error-estimate of $m$.

Optimum Rule: Given the received segment $r=\rho$, take the estimate of the sync word location $m$ to be the value of $\mu$, $0 \leqslant \mu \leqslant N-1$, which maximizes the statistic

$$
S_{O}=\sum_{i=0}^{L-1} s_{i} \rho_{i+\mu}-\sum_{i=0}^{L-1} f\left(\rho_{i+\mu}\right)
$$

where

$$
f(x)=\frac{N_{0}}{2 \sqrt{E}} \ln \cosh \left(\frac{2 \sqrt{E} x}{N_{0}}\right) .
$$

(The additions in the subscripts of (2) are taken to be modulo $N$.)

It has been common engineering practice to disregard the second term in (2) and base the decision on the correlation term only. We state this rule specifically.

Correlation Rule: Choose $\mu$ to maximize

$$
S_{C}=\sum_{i=0}^{L-1} s_{i} \rho_{i+\mu}
$$

For very high and very low SNR's the function (3) may be approximated closely by much simpler expressions, leading to the following rules.

Optimum Rule for High SNR: Choose $\mu$ to maximize

$$
S_{H}=\sum_{i=0}^{L-1} s_{i} \rho_{i+\mu}-\sum_{i=0}^{L-1}\left|\rho_{i+\mu}\right|
$$

Optimum Rule for Low SNR: Choose $\mu$ to maximize

$$
S_{L}=\sum_{i=0}^{L-1} s_{i} \rho_{i+\mu}-\frac{\sqrt{E}}{N_{0}} \sum_{i=0}^{L-1} \rho_{i+\mu}^{2} .
$$

From this brief summary of previous results we turn to a discussion of the performance attainable with each of the previous decision rules.

\section{SYNCHRONIZER PERFORMANCE}

There are essentially two mechanisms that may cause the synchronizer to choose an incorrect value of $\mu$. Since no restrictions have been imposed on the data it is possible for the sync pattern to appear elsewhere than its intended location in which case there is a large probability that the synchronizer makes a mistake, even when the noise is insignificantly small. As we shall shortly see, we can analyze this situation to obtain an exact explicit expression for the performance in the limiting case of high SNR's. As noise increases, the dissimilarity between the sync word and any data segment will tend to decrease and the performance deteriorates quite rapidly. Unfortunately, this mechanism is complicated enough to escape even an approximate analysis, and we must stick to computer simulations to determine the performance in this range.

It is generally desirable to choose a sync word with good autocorrelation properties, and in particular a sync word $s=\left(s_{0}, s_{1}, \cdots, s_{L-1}\right)$ should always satisfy the condition

$$
\begin{aligned}
\left(s_{0}, s_{1}, \cdots, s_{l-1}\right) \neq\left(s_{L-l}, s_{L-l+1}, \cdots,\right. & \left.s_{L-1}\right), \\
& l=1,2, \cdots, L-1
\end{aligned}
$$


to ensure that no overlap between a sync word and random data bits may equal the sync word itself. If (7) is not satisfied, the pattern is effectively shortened. We now analyze the optimum synchronizer performance in the noise-free case, assuming that (7) holds true.

Let $D_{i}$ denote the total number of distinct $N-L$ bit data sequences in which $s$ is imitated exactly $i$ times, $0 \leqslant i \leqslant$ $M \triangleq\lfloor N-L / L\rfloor$. We then have

$$
D_{M}=\left(\begin{array}{c}
N-L-(L-1) M \\
M
\end{array}\right) 2^{N-L-L M}
$$

where the binomial coefficient is the number of ways in which the $M$ patterns may be mixed with the remaining $N-L-L M$ random bits. Next we observe that

$D_{M-1}=\left(\begin{array}{c}N-L-(L-1)(M-1) \\ M-1\end{array}\right) 2^{N-L-L(M-1)}-\left(\begin{array}{c}M \\ M-1\end{array}\right) D_{M}$

where the first term is obtained as above by fixing $M-1$ patterns, leaving the remaining $N-L-L(M-1)$ data bits random, while the second term removes those of the sequences counted this way, which contain even more than $M-1$ patterns. The form of the correction term follows easily from noting that there are $\left(\begin{array}{c}M \\ M-1\end{array}\right)$ ways of placing $M-1$ patterns such that a cer tain combination of the remaining random bits will produce a given sequence containing $M$ patterns.

By a trivial extension of the above arguments we get the general expression,

$$
D_{i}=\left(\begin{array}{c}
N-L-(L-1) i \\
i
\end{array}\right) 2^{N-L-L i}-\sum_{j=i+1}^{M}\left(\begin{array}{l}
j \\
i
\end{array}\right) D_{j}
$$

Multiplying both sides of $(10)$ by $(-1)^{i+1} /(i+1)$ and summing over $i$, we obtain

$$
\begin{aligned}
\sum_{i=1}^{M}(-1)^{i+1} \frac{1}{i+1}\left(\begin{array}{c}
N-L-(L-1) i \\
i
\end{array}\right) 2^{N-L-L i} \\
\quad=\sum_{i=1}^{M} \sum_{j=i}^{M}(-1)^{i+1} \frac{1}{i+1}\left(\begin{array}{c}
j \\
i
\end{array}\right) D_{j} \\
=\sum_{j=1}^{M} \sum_{i=1}^{j}(-1)^{i+1} \frac{1}{i+1}\left(\begin{array}{l}
j \\
i
\end{array}\right) D_{j} \\
=\sum_{j=1}^{M} \sum_{i=1}^{j}(-1)^{i+1} \frac{1}{j+1}\left(\begin{array}{c}
j+1 \\
i+1
\end{array}\right) D_{j} \\
=\sum_{j=1}^{M} \frac{1}{j+1} \sum_{i=2}^{j+1}(-1)^{i}\left(\begin{array}{c}
j+1 \\
i
\end{array}\right) D_{j} \\
=\sum_{j=1}^{M} \frac{j}{j+1} D_{j} .
\end{aligned}
$$

Now, let $P\left(\epsilon_{S}\right)$ denote the probability of having a sync error, i.e., the probability that the synchronizer decides for an incorrect value of $\mu$. In the noise-free case the synchronizer must select at random between all the positions where a correct pattern begins, so we have the relation

$$
P\left(\epsilon_{s}\right)=\sum_{j=1}^{M} \frac{j}{j+1} 2^{-(N-L)} D_{j}
$$

since $2^{-(N-L)} D_{j}$ is the probability of having $j+1$ patterns (including the one that was intentionally inserted) in an $N$-bit frame. Substituting (11) into (12) we immediately have the desired closed-form solution for $P\left(\epsilon_{s}\right)$,

$$
P\left(\epsilon_{s}\right)=\sum_{i=1}^{M}(-1)^{i+1} \frac{1}{i+1}\left(\begin{array}{c}
N-L-(L-1) i \\
i
\end{array}\right) 2^{-L i}
$$

Note that for high SNR's the asymptotic performance (13) is attained with either of the statistics $S_{O}, S_{C}$, and $S_{H}$. However, when $S_{L}$ is used, it is evident from (6) that for large values of $E / N_{0}$ the second term in $S_{L}$ will dominate, resulting in a completely random choice between the $N$ possible values of $\mu$ so that for this statistic we have $P\left(\epsilon_{S}\right)=(N-1) / N$ when noise is absent.

Computer simulations were carried out to investigate the performance of the four synchronizer statistics in the presence of noise. . For each signal-to-noise ratio 1000 frames were processed by the four synchronizers simultaneously such that for each choice of frame length and sync word we obtained four curves showing the relative number of frames with false synchronization versus $E / N_{0}$ for each of the four synchronizer statistics $S_{O}, S_{C}, S_{H}$, and $S_{L}$. Results are shown in Figs. 1 and 2 for $(N, L)=(28,7)$ and $(N, L)=(91,13)$, respectively. The sync words used in these simulations were the 7-b Barker sequence $(1,1,1,-1,-1,1,-1)$ and the 13-b NeumanHofman sequence $(1,1,1,1,1,1,-1,-1,1,1,-1,1,-1)$ both of which were also considered by Massey [1] (the 13-b Barker code also investigated in [1] does not satisfy the condition (7) and it is clearly a poor choice for a sync pattern). In both figures the asymptotic values of $P\left(\epsilon_{s}\right)$ are shown as calculated from (13).

The critical observation to be made from the simulation results is that the statistic $S_{H}$ (optimum for high SNR's) is only a fraction of a decibel inferior to the optimum statistic $S_{O}$ throughout the investigated range, i.e., for all SNR's down to $-3 \mathrm{~dB}$ and less. The pure correlation $S_{C}$ performs significantly worse, the difference amounting to as much as $6 \mathrm{~dB}$ in a range of great practical importance. The 3-dB difference reported by Massey applies to a narrow range of SNR's near $0 \mathrm{~dB}$, but is in no way general. Observe also that the statistic $S_{L}$ is of no practical value whatsoever. The near-optimality of $S_{H}$ over the entire range was further verified by running a few simulations with a 3-b and a 26-b sync word, in both cases with results very much analogous to those shown in Figs. 1 and 2 .

The statistic $S_{H}$ is extremely simple to instrument and it definitely is to be preferred for most practical applications. Besides being a close approximation to $S_{O}$ for high SNR's, $S_{H}$ has another interesting interpretation as well. The frame synchronization problem as stated here may be viewed as $N$-ary detection in additive white Gaussian noise, where the $N$ signals $\sqrt{E} T^{m}(s d)$ are known except for the random vector $d$. The so-called "generalized likelihood ratio test" [2] is a useful although generally suboptimum tool for solving this type of problem. Under each hypothesis the receiver forms a maximum likelihood (ML) estimate of the unknown vector $d$ and uses this estimate $\hat{d}$ in a conventional correlation detection scheme just as if it were correct. In the present system, for $m=\mu$, we have

$$
\hat{d}_{i}=\frac{\left|\rho_{i+\mu}\right|}{\rho_{i+\mu}}
$$

such that the correlation between $\rho$ and the normalized signal $T^{\mu}(s \hat{d})$ becomes

$\sum_{i=0}^{L-1} s_{i} \rho_{i+\mu}+\sum_{i=L}^{N-1}\left|\rho_{i+\mu}\right|=\sum_{i=0}^{L-1} s_{i} \rho_{i+\mu}-\sum_{i=0}^{L-1}\left|\rho_{i+\mu}\right|+\sum_{i=0}^{N-1}\left|\rho_{i+\mu}\right|$ 


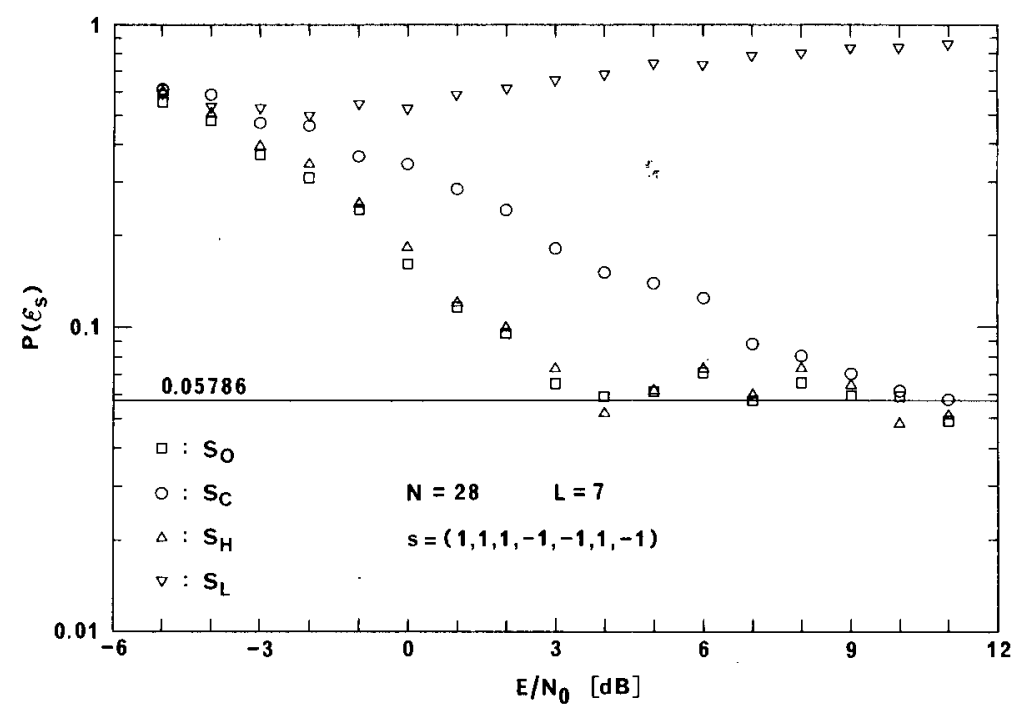

Fig. 1. Probability of false synchronization versus $E / N_{0}$ for each of the four statistics $S_{O}, S_{C}, S_{H}$, and $S_{L}$. Frame format $(N, L)=(28,7)$. Sync word $(1,1,1,-1,-1,1,-1)$.

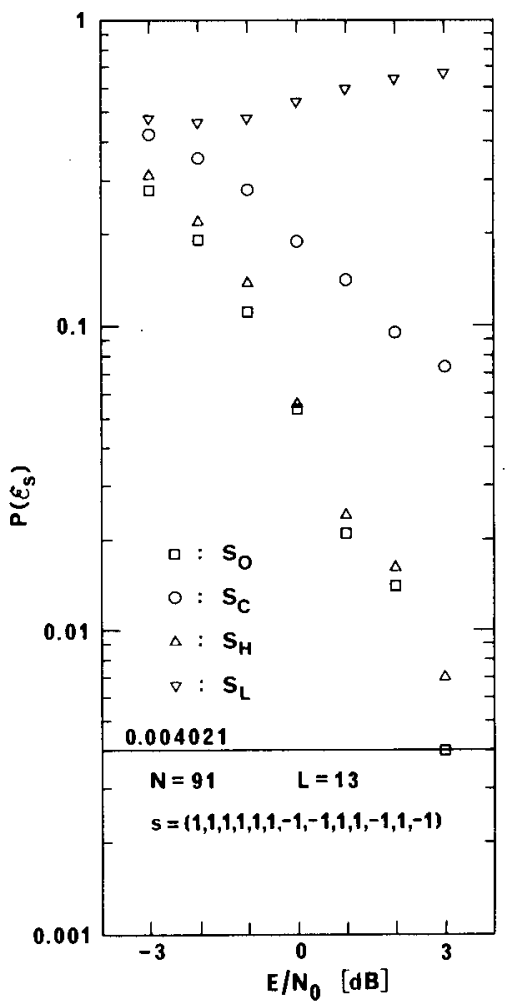

Fig. 2. Probability of false synchronization versus $E / N_{0}$ for each of the four statistics $S_{O}, S_{C}, S_{H}$, and $S_{L}$. Frame format $(N, L)=(91,13)$. Sync word $(1,1,1,1,1,1,-1,-1,1,1,-1,1,-1)$.

To see that the statistic (15) is equivalent to $S_{H}$ we need only observe that the last summation in (15) is independent of $\mu$ and hence may be discarded.

\section{CONCLUSION}

By far the most significant result of the work reported here is the discovery that a frame synchronizer using the statistic $S_{H}$ yields virtually optimum performance for all signal-tonoise ratios of practical interest. While implementation of the optimum synchronizer would require the awkward func- tion (3) to be instrumented, $S_{H}$ is just as easy (in certain cases even faster) to calculate as the pure correlation $S_{C}$. We also noted that the loss encountered by using $S_{C}$ instead of $S_{H}$ may be quite severe (up to about $6 \mathrm{~dB}$ ) for certain signal-tonoise ratios of great practical interest; but in the limit as the noise disappears their performance become identical. An expression was given for the probability of false synchronization in the absence of noise.

\section{REFERENCES}

[1] J. L. Massey "Optimum frame synchronization," IEEE Trans. Commun., vol. COM-20, pp. 115-119, Apr. 1972.

[2] H. L. van Trees, Detection, Estimation, and Modulation Theory: Part I. New York: Wiley, 1968, pp. 86-96.

\section{Pseudonoise Code Acquisition Using Majority Logic Decoding}

\section{CHARLES C. KILGUS}

Abstract-This paper considers the use of majority logic decoding as a pseudonoise code acquisition technique. A bound on the probability of code acquisition is derived and it is shown that the probability of acquiring an 8191 code in one attempt can be made nearly one at -10-dB SNR.

\section{INTRODUCTION}

The maximal length shift-register codes are perhaps the best known pseudonoise (PN) codes, i.e., codes with a two level autocorrelation function [1]. PN codes have found application in ranging and communication systems, e.g., in spacecraft ranging applications the output of a continuously cycling shiftregister generator is relayed through a transponder on the spacecraft and recovered on the ground. The delay between the transmitted and received code provides a measure of the range to the spacecraft.

The codes are usually recovered by correlation with a

Paper approved by the Communication Theory Committee of the Communications Society without oral presentation. Manuscript received June 13,1972 .

The author is with the Applied Physics Laboratory, the Johns Hopkins University, Silver Spring, Md.

$$
\text { ALSO ICC'73, PP. 23.16-23.17 }
$$

